\title{
QUASI-LIPSCHITZ EQUIVALENCE OF SUBSETS OF AHLFORS-DAVID REGULAR SETS
}

\author{
Qiuli Guo, Hao Li and Qin Wang* \\ Zhejiang Wanli University, Institute of Mathematics \\ Ningbo, Zhejiang, 315100, P. R. China; guoqiuli@zwu.edu.cn \\ Zhejiang Wanli University, Institute of Mathematics \\ Ningbo, Zhejiang, 315100, P. R. China; kevinlee9809@hotmail.com \\ Zhejiang Wanli University, School of Computer Science and Information Technology \\ Ningbo, Zhejiang, 315100, P. R. China; qinwang@126.com
}

\begin{abstract}
In the paper, it is proved that for any Ahlfors-David $s$-regular sets $E$ and $F$ in Euclidean spaces, there exist subsets $E^{\prime} \subset E$ and $F^{\prime} \subset F$ such that $\operatorname{dim}_{H} E^{\prime}=\operatorname{dim}_{H} F^{\prime}=s$ and $E^{\prime}, F^{\prime}$ are quasi-Lipschitz equivalent.
\end{abstract}

\section{Introduction}

For $E \subset \mathbf{R}^{n}$ and $F \subset \mathbf{R}^{m}$, a bijection $f: E \rightarrow F$ is said to be bilipschitz if there is a positive number $L$ such that

$$
L^{-1}|x-y| \leq|f(x)-f(y)| \leq L|x-y| \text { for all } x, y \in E .
$$

We say that sets $E$ and $F$ in Euclidean spaces are bilipschitz equivalent if there exists a bilipschitz bijection from $E$ onto $F$ and denote by $E \sim F$. We say that $E$ can be bilipschitz embedded into $F$ if there exists a subset $F^{\prime}$ of $F$ such that $E \sim F^{\prime}$ and denote by $E \hookrightarrow F$.

Definition 1. [8] A compact set $F$ is said to be Ahlfors-David s-regular ( $s$ regular for short), if there is a Borel measure $\nu$ supported on $E$ and a constant $C_{F}$ such that

$$
C_{F}^{-1} r^{s} \leq \nu(B(x, r)) \leq C_{F} r^{s}
$$

for all $x \in F$ and $0<r \leq|F|$, where $|F|$ is the diameter of $F$ and $B(x, r)$ is the closed ball with center $x$ and radius $r$.

Remark 1. Any $s$-regular set has Hausdorff dimension $s$.

Remark 2. Any $C^{1+\gamma}(\gamma>0)$ self-conformal set $F$ satisfying the open set condition is $s$-regular, where $s=\operatorname{dim}_{H} F$ and $\nu=\left.\mathcal{H}^{s}\right|_{F}$. In particular, any self-similar set satisfying the open set condition is regular.

Suppose that $A$ and $B$ are regular with $\operatorname{dim}_{H} A<\operatorname{dim}_{H} B$. Mattila and Saaranen [9] proved that for any $\epsilon>0$, there exists a regular subset $A^{\prime}$ of $A$ with $\mid \operatorname{dim}_{H} A^{\prime}-$ $\operatorname{dim}_{H} A \mid<\epsilon$ such that $A^{\prime} \hookrightarrow B$, where $A^{\prime}$ is bilipschitz equivalent to a generalized

doi:10.5186/aasfm.2014.3930

2010 Mathematics Subject Classification: Primary 28A80.

Key words: Fractal, Ahlfors-David regularity, quasi-Lipschitz equivalence, Moran set.

${ }^{*}$ Corresponding author. This work is supported by National Natural Science of Foundation of China (Nos. 11371329, 11071224), NCET and NSF of Zhejiang Province (Nos. LR13A1010001, LY12F02011). 
Cantor set, which is self-similar. They also obtained that if $\operatorname{dim}_{H} A<1$, then $A \hookrightarrow B$. However, for $\operatorname{dim}_{H} A=1$, Deng etc. [2] pointed out that if $A=[0,1]$, any subset $A^{\prime} \subset[0,1]$ with positive Lebesgue measure can not be bilipschitz embedded into any self-similar set satisfying the strong separation condition (SSC).

The above works raise the following question: For two regular subsets $A$ and $B$ of Euclidean spaces satisfying $\operatorname{dim}_{H} A=\operatorname{dim}_{H} B$, what kind of good subsets $A^{\prime}$ of $A$ can be bilipschitz embedded into $B$ ? Here we hope that the good subset $A^{\prime}$ is close to $A$, for example, $\left|\operatorname{dim}_{H} A^{\prime}-\operatorname{dim}_{H} A\right|$ is small enough or $\mathcal{H}^{s}\left(A^{\prime}\right)>0$ with $s=\operatorname{dim}_{H} A$.

Llorente and Mattila [7] assumed open set condition and then proved that for selfconformal sets $E$ and $F$ with the same dimension $s$, if there exist subsets $E^{\prime} \subset E$ and $F^{\prime} \subset F$ with $\mathcal{H}^{s}\left(E^{\prime}\right), \mathcal{H}^{s}\left(F^{\prime}\right)>0$ such that $E^{\prime} \sim F^{\prime}$, then $E \sim F$. For self-similar sets with the same dimension satisfying SSC, Deng etc. [2] obtained the similar result. However, Falconer and Marsh [3] pointed out that the self-similar sets (satisfying $\mathrm{SSC}$ ) with the same dimension need not be bilipschitz equivalent. Then the results of $[7,2]$ imply that for two self-similar sets with $\operatorname{dim}_{H} E=\operatorname{dim}_{H} F=s$ but $E \not F$, we can not find subsets $E^{\prime}(\subset E), F^{\prime}(\subset F)$ with positive $\mathcal{H}^{s}$ measure such that $E^{\prime} \sim F^{\prime}$.

We will introduce a notion weaker than bilipschitz equivalence.

Definition 2. [18] The compact subsets $E$ and $F$ of Euclidean spaces are said to be quasi-Lipschitz equivalent, if there is a bijection $f: E \rightarrow F$ such that for all $x_{1}, x_{2} \in E$,

$$
\frac{\log \left|f\left(x_{1}\right)-f\left(x_{2}\right)\right|}{\log \left|x_{1}-x_{2}\right|} \rightarrow 1 \text { uniformly as }\left|x_{1}-x_{2}\right| \rightarrow 0 .
$$

We say that $E$ can be quasi-Lipschitz embedded into $F$ if $E$ is quasi-Lipschitz equivalent to a subset of $F$.

Remark 3. It is proved in [18] that two self-conformal sets $E, F$ satisfying SSC are quasi-Lipschitz equivalent if and only if they have the same Hausdorff dimension. This result fails for bilipschitz equivalence, e.g. self-similar sets satisfying SSC as shown in [3, 19]. [5] and [12] discussed the quasi-Lipschitz equivalence of Moran sets and regular sets.

This paper focuses an alternative question: For regular sets $A$ and $B$ in Euclidean spaces with $\operatorname{dim}_{H} A=\operatorname{dim}_{H} B$, what kinds of good subsets of $A$ can be quasiLipschitz embedded into $B$ ?

Now we give our main theorem.

Theorem 1. Suppose that $s>0$. For $s$-regular sets $E$ and $F$ in Euclidean spaces, there exist subsets $E^{\prime} \subset E$ and $F^{\prime} \subset F$ with $\operatorname{dim}_{H} E^{\prime}=\operatorname{dim}_{H} F^{\prime}=s$ such that $E^{\prime}$ and $F^{\prime}$ are quasi-Lipschitz equivalent.

Frostman's lemma shows that if $E \subset \mathbf{R}^{d}$ is compact and $\mathcal{H}^{t}(E)>0$, then there is a Borel measure $\mu$ supported on $E$ such that

$$
\mu(B(x, r)) \leq r^{t}
$$

for all $x \in \mathbf{R}^{d}, r>0$. Let $E^{\prime}$ be the support of the above measure $\mu$. Can we obtain a constant $c>0$ such that

$$
c r^{t} \leq \mu(B(x, r)) \leq r^{t}
$$


for all $x \in E^{\prime}$ and $r \leq\left|E^{\prime}\right|$ ? If inequality (1.4) holds, then $E$ contains an AhlforsDavid $t$-regular subset $E^{\prime}$.

Then a natural question is whether $E$ with $\operatorname{dim}_{H} E=s$ always contains a t-regular subset with $t \in(0, s]$. The following proposition offers a negative answer.

Proposition 1. For any given $s \in(0,1)$, there exists an $s$-Hausdorff dimensional Moran set $F \subset \mathbf{R}^{1}$ such that $F$ does not contain any regular subset.

For $s=1$, Example 5.3 in [9] gave a set with positive $\mathcal{L}^{1}$ measure which contains no regular subset. In fact, the key point is that the set in [9] does not contain any uniformly perfect subset. Inspired by this, for any given $s \in(0,1)$, we will obtain a Moran set $[13,14]$ with the structure $\left(I,\left\{n_{k}\right\},\left\{c_{k}\right\}\right)$, where $I$ is the closed interval $[0,1], n_{k} \rightarrow \infty$ and $c_{k}=n_{k}^{-1 / s}$. Then this Moran set, with $s$-Hausdorff dimension, contains no regular subsets. In fact, it is the key that none of its subsets can be uniformly perfect.

When is a Moran set Ahlfors-David regular? We note that the above Moran set

$$
c_{*}=\inf _{k} c_{k}=0 \text {, }
$$

where $c_{k, 1}=\cdots=c_{k, n_{k}}=c_{k}$. For the Moran set with structure $\left(J,\left\{n_{k}\right\}_{k \geq 1},\left\{c_{k, j}\right\}_{k \geq 1}\right.$, $j \leq n_{k}$ ) $[13,14]$, under the condition

$$
c_{*}=\inf _{k, j} c_{k, j}>0
$$

the following Proposition 2 gives a necessary and sufficient condition for a Moran set on $\mathbf{R}^{1}$ to be regular.

Proposition 2. Suppose a Moran set $F$ is defined as in (2.4) on $\mathbf{R}^{1}$ satisfying that $c_{*}=\inf _{k, j} c_{k, j}>0$. Then $F$ is s-regular if and only if there are constants $0<\alpha, \beta<\infty$ such that

$$
\alpha \leq \prod_{k=1}^{N} \sum_{j=1}^{n_{k}} c_{k, j}^{s} \leq \beta \text { for all } N>0 .
$$

Remark 4. For the Moran set in the proof of Proposition 1 , let $c_{i, 1}=\cdots=$ $c_{i, n_{i}}=c_{i}$ for all $i$, we have $\prod_{k=1}^{N} \sum_{j=1}^{n_{k}} c_{k, j}^{s}=1$ for all $k$. Then the condition $c_{*}>0$ is necessary.

The paper is organized as follows. In Section 2, we prove Theorem 1 by constructing a special homogeneous Moran subset, which is quasi Ahlfors-David s-regular and quasi uniformly disconnected. The proof is based on Lemma 1 from [11]. In Section 3, we prove Proposition 1 using uniform perfectness [10] and Proposition 2 using the measure in $[1,6]$.

\section{Moran subsets with full dimension}

2.1. Moran sets. Suppose that $J \subset \mathbf{R}^{d}$ is a compact set with nonempty interior. Let $\left\{n_{k}\right\}_{k \geq 1}$ be a given positive integer sequence satisfying $n_{k} \geq 2$ for all $k$. Let $\psi=\psi_{k}$ be a finite positive real vector sequence, where

$$
\psi_{k}=\left(c_{k, 1}, \cdots, c_{k, n_{k}}\right), \quad 0<c_{k, j}<1, k \in \mathbf{N}, 1 \leq j \leq n_{k} .
$$

The set of finite words is denoted by $\mathcal{D}^{\infty}=\bigcup_{k=0}^{\infty} \mathcal{D}^{k}$, where

$$
\mathcal{D}^{k}=\left\{i_{1} \cdots i_{k}: i_{j} \in \mathbf{N} \cap\left[1, n_{j}\right] \text { for all } j\right\}
$$


and $D^{0}=\{\emptyset\}$ and $\emptyset$ is the empty word. Given $\sigma=i_{1} \cdots i_{k} \in \mathcal{D}^{k}, \tau=j_{1} \cdots j_{l} \in \mathcal{D}^{l}$, denote the word $\sigma * \tau=i_{1} \cdots i_{k} j_{1} \cdots j_{l}$. The length of the word $\sigma \in \mathcal{D}^{k}$ is denoted by $|\sigma|(=k)$.

We say that the family $\mathcal{F}=\left\{J_{\sigma}: \sigma \in \mathcal{D}^{\infty}\right\}$ of subsets of $\mathbf{R}^{d}$ has Moran structure, if the following three conditions hold:

(i) for any $\sigma \in \mathcal{D}^{\infty}, J_{\sigma}$ is geometrically similar to $J$, where we denote by $J_{\emptyset}=J$;

(ii) for any $k \geq 0$ and $\sigma \in \mathcal{D}^{k-1}$,

satisfying

$$
J_{\sigma * 1}, \cdots, J_{\sigma * n_{k}} \subset J_{\sigma}
$$

$\operatorname{int}\left(J_{\sigma * i}\right) \cap \operatorname{int}\left(J_{\sigma * j}\right)=\varnothing \quad$ whenever $i \neq j$

where int denotes the interior of the set;

(iii) for any $k \geq 1, \sigma \in \mathcal{D}^{k-1}$ and $1 \leq j \leq n_{k}$, it holds that

$$
\frac{\left|J_{\sigma * j}\right|}{\left|J_{\sigma}\right|}=c_{k, j}
$$

Then we call the following compact set

$$
F=\bigcap_{k=0}^{\infty} \bigcup_{\sigma \in \mathcal{D}^{k}} J_{\sigma}
$$

a Moran set in $\mathbf{R}^{d}$ with the structure $\left(J,\left\{n_{k}\right\},\left\{\psi_{k}\right\}\right)=\left(J,\left\{n_{k}\right\},\left\{c_{k, j}\right\}\right)$. The members of the family $\left\{J_{\sigma}: \sigma \in \mathcal{D}^{k}\right\}$ are called basic elements of rank $k$.

A Moran set $F$ defined in (2.4) is said to be homogeneous with the structure $\left(J,\left\{n_{k}\right\},\left\{c_{k}\right\}\right)$, if $c_{k, 1}=\cdots=c_{k, n_{k}}=c_{k}$ for any $k \geq 1$.

When we talk about a Moran set on $\mathbf{R}^{1}$, for convenience as in $[13,14]$, we always assume that the initial set $J$ is a closed interval. The members of the family $\left\{J_{\sigma}: \sigma \in\right.$ $\left.\mathcal{D}^{k}\right\}$ are called basic intervals of rank $k$.

2.2. Result on quasi-Lipschitz equivalence. Recall the notions of quasi uniform disconnectedness and quasi Ahlfors-David regularity in [11].

Definition 3. We say that a subset $F$ of metric space $X$ is quasi uniformly disconnected if there is a function $\rho:(0, \infty) \rightarrow(0, \infty)$ with $\lim _{t \rightarrow 0} \frac{\log \rho(t)}{\log t}=1$ such that for any $x \in F, r>0$, there is a subset $B \subset F$ such that

$$
F \cap B(x, \rho(r)) \subset B \subset B(x, r) \text { and } \operatorname{dist}(B, F \backslash B)>\rho(r),
$$

where $\operatorname{dist}\left(A_{1}, A_{2}\right)$ denotes the least distance between $A_{1}$ and $A_{2}$.

Definition 4. A compact set $F$ is said to be quasi Ahlfors-David s-regular, if there exists a Borel measure $\nu$ supported on $F$ and a non-decreasing function $h:(0,|F|) \rightarrow(0,+\infty)$ with $\lim _{t \rightarrow 0} h(t)=0$, such that for all $x \in F$ and $0<r \leq|F|$,

$$
s(1-h(r)) \leq \frac{\log \nu(B(x, r))}{\log r} \leq s(1+h(r)) .
$$

In fact, any quasi $s$-regular set has Hausdorff dimension $s$. Inequality (2.6) means that as $r \rightarrow 0$,

$$
\frac{\log \nu(B(x, r))}{\log r} \rightarrow s \text { uniformly for all } x \in F .
$$

The reference [11] points out the following result on quasi-Lipschitz equivalence. 
Lemma 1. Suppose $A$ and $B$ are compact and quasi uniformly disconnected in metric spaces. If $A$ and $B$ are quasi s-regular and quasi $t$-regular respectively, then they are quasi-Lipschitz equivalent if and only if $s=t$.

2.3. Construction of Moran subsets. We will construct subsets of full dimension and obtain their quasi-Lipschitz equivalence by using Lemma 1 .

Suppose that $E \subset \mathbf{R}^{d}$ is an $s$-regular set with the measure $\nu$ supported on $E$ such that

$$
C_{E}^{-1} r^{s} \leq \nu(B(x, r)) \leq C_{E} r^{s}
$$

for all $x \in E$ and $0<r \leq|E|$, where $C_{E}>0$ is a constant. Now, we will construct recursively a full dimensional homogeneous Moran subset $E^{\prime}$ of $E$ such that $E^{\prime}$ is quasi $s$-regular and quasi uniformly disconnected.

Given $\varepsilon>0$ small enough, let $R_{0}=1$ and

$$
R_{k}=\varepsilon^{1+2+\cdots+k} \text { for all } k \geq 1 \text {. }
$$

Then

$$
\frac{R_{k}}{R_{k-1}}=\varepsilon^{k} \rightarrow 0 \text { and } \frac{\log R_{k}}{\log R_{k-1}} \rightarrow 1 \text { as } k \rightarrow \infty .
$$

For any compact subset $A$ of $\mathbf{R}^{d}$, let $M_{\varepsilon}(A)$ and $N_{\varepsilon}(A)$ be the maximum number of disjoint $\varepsilon$-balls with centers in $A$ and the minimum number of $\varepsilon$-balls needed to cover $A$ respectively. By [14], we have

$$
C_{d} N_{\varepsilon}(A) \leq N_{2 \varepsilon}(A) \leq M_{\varepsilon}(A) \leq N_{\varepsilon}(A),
$$

where $C_{d}>0$ is a constant depending on the space $\mathbf{R}^{d}$.

Fix $x_{\emptyset} \in E$ for empty word $\emptyset$. Since $\varepsilon$ is small enough, we can take $n_{1}=2$ and $x_{1}, x_{2} \in B\left(x_{\emptyset}, 1 / 2\right) \cap E$ such that $B\left(x_{1}, \varepsilon\right) \cap B\left(x_{2}, \varepsilon\right)=\varnothing$.

By induction, assume we obtain points $\left\{x_{i_{1} \cdots i_{k-1}}\right\}_{i_{1} \cdots i_{k-1}} \subset E$ satisfying

(1) $x_{i_{1} \cdots i_{k-2} i_{k-1}} \in B\left(x_{i_{1} \cdots i_{k-2}}, R_{k-2} / 2\right) \cap E$ for all $i_{1} \cdots i_{k-2} i_{k-1}$;

(2) $B\left(x_{i_{1} \cdots i_{k-2} i_{k-1}}, R_{k-1}\right) \cap B\left(x_{i_{1} \cdots i_{k-2} j_{k-1}}, R_{k-1}\right)=\varnothing$ if $i_{k-1} \neq j_{k-1}$.

Given point $x:=x_{i_{1} \cdots i_{k-1}} \in E$, suppose that $\left\{B\left(y_{i}, R_{k} / 2\right)\right\}_{i=1}^{N_{k}(x)}$ is a covering of $B\left(x, R_{k-1} / 2\right) \cap E$, where $y_{i} \in B\left(x, R_{k-1} / 2\right)$ and $N_{k}(x):=N_{R_{k} / 2}\left(B\left(x, R_{k-1} / 2\right) \cap E\right)$. Using the definition of $N_{\varepsilon}(\cdot)$, we can take $z_{i} \in B\left(x, R_{k-1} / 2\right) \cap E$ such that

$$
B\left(x, R_{k-1} / 2\right) \cap E \subset \bigcup_{i=1}^{N_{k}(x)} B\left(z_{i}, R_{k}\right) .
$$

Therefore, we have

$$
\begin{aligned}
2^{-s} \cdot C_{E}^{-1} \cdot R_{k-1}^{s} & \leq \nu\left(B\left(x, R_{k-1} / 2\right) \cap E\right) \leq \nu\left(\bigcup_{i=1}^{N_{k}(x)} B\left(z_{i}, R_{k}\right)\right) \\
& \leq \sum_{i=1}^{N_{k}(x)} \nu\left(B\left(z_{i}, R_{k}\right)\right)=N_{k}(x) \cdot C_{E} \cdot R_{k}^{s} .
\end{aligned}
$$

Let $M_{k}(x):=M_{R_{k}}\left(B\left(x, R_{k-1} / 2\right) \cap E\right)$. It follows from (2.9) that

$$
M_{k}(x) \geq C_{d}^{2} N_{k}(x)
$$

which implies

$$
M_{k}(x) \geq D \cdot\left(R_{k-1} / R_{k}\right)^{s}=D \cdot \varepsilon^{-k s},
$$

where $D=C_{d}^{2} \cdot 2^{-s} \cdot C_{E}^{-2}$. 
Therefore, by (2.10) we can take $n_{k}=\left[D \varepsilon^{-k s}\right]$ points

$$
\left\{x_{i_{1} \cdots i_{k-1} i_{k}}\right\}_{i_{k}=1}^{n_{k}} \subset B\left(x_{i_{1} \cdots i_{k-1}}, R_{k-1} / 2\right) \cap E
$$

satisfying

$$
B\left(x_{i_{1} \cdots i_{k-1} i_{k}}, R_{k}\right) \cap B\left(x_{i_{1} \cdots i_{k-1} j_{k}}, R_{k}\right)=\varnothing \text { for any } i_{k} \neq j_{k},
$$

where $[a]$ is the integral part of $a$. Then

$$
E^{\prime}=\bigcap_{k \geq 1} \bigcup_{i_{1} \cdots i_{k}} B\left(x_{i_{1} \cdots i_{k}}, R_{k}\right) .
$$

is a homogeneous Moran subset of $E\left(\subset \mathbf{R}^{d}\right)$ with structure $\left(B\left(x_{\emptyset}, 1\right),\left\{n_{k}\right\},\left\{c_{k}\right\}\right)$ where

$$
n_{1}=2, n_{k}=\left[D \varepsilon^{-k s}\right] \text { for } k \geq 2 \text { and } c_{k}=R_{k} / R_{k-1}=\varepsilon^{k} \text {. }
$$

2.4. The proof of Theorem 1. In fact, for any $x \in B\left(x_{i_{1} \cdots i_{k} i_{k+1}}, R_{k+1}\right)$, we have

$$
\left|x-x_{i_{1} \cdots i_{k}}\right| \leq R_{k} / 2+R_{k+1} .
$$

Given $i_{1} \cdots i_{k} \neq j_{1} \cdots j_{k}$, applying (2.14) we have

$$
\begin{aligned}
& B\left(x_{i_{1} \cdots i_{k} i_{k+1}}, R_{k+1}\right) \subset B\left(x_{i_{1} \cdots i_{k}}, R_{k} / 2+R_{k+1}\right) \text { for all } i_{k+1}, \\
& B\left(x_{j_{1} \cdots j_{k} j_{k+1}}, R_{k+1}\right) \subset B\left(x_{j_{1} \cdots j_{k}}, R_{k} / 2+R_{k+1}\right) \text { for all } j_{k+1} .
\end{aligned}
$$

We can take small $\varepsilon$ in $(2.7)$ such that $\frac{1}{2} \varepsilon^{k}+\varepsilon^{k+(k+1)}<1$ and $\varepsilon^{k+1}<\frac{1}{6}$ for all $k \geq 1$, which implies

$$
R_{k-1}>R_{k} / 2+R_{k+1} \text { and } R_{k}>6 R_{k+1} .
$$

Let $\left.B=B\left(x_{i_{1} \cdots i_{k}}, R_{k} / 2+R_{k+1}\right)\right)$. Since $B\left(x_{i_{1} \cdots i_{k}}, R_{k}\right) \cap B\left(x_{j_{1} \cdots j_{k}}, R_{k}\right)=\varnothing$, using (2.15) we have

$$
\operatorname{dist}\left(B, E^{\prime} \backslash B\right) \geq 2\left(R_{k}-\left(R_{k} / 2+R_{k+1}\right)\right)>2 R_{k+1} .
$$

Now, according to Lemma 1, we will check the properties of $E^{\prime}$.

Lemma 2. $E^{\prime}$ is quasi uniformly disconnected.

Proof. Suppose that $2 R_{k-1}<r \leq 2 R_{k-2}$ and $x \in B\left(x_{i_{1} \cdots i_{k} i_{k+1}}, R_{k+1}\right) \cap E^{\prime}$. Let $\rho(r)=2 R_{k+1}$.

We take $B=E^{\prime} \cap B\left(x_{i_{1} \cdots i_{k}}, R_{k} / 2+R_{k+1}\right)$ as above. Using (2.16), we have

$$
E^{\prime} \cap B\left(x, 2 R_{k+1}\right) \subset B .
$$

Since $\left|x-x_{i_{1} \cdots i_{k}}\right| \leq R_{k} / 2+R_{k+1}$ and $R_{k-1}>R_{k} / 2+R_{k+1}$, we have

$$
B \subset B\left(x, 2 R_{k-1}\right) \subset B(x, r) .
$$

By (2.8), we note that

$$
1 \leftarrow \frac{\log \left(2 R_{k+1}\right)}{\log \left(2 R_{k-1}\right)} \leq \frac{\log \rho(r)}{\log r} \leq \frac{\log \left(2 R_{k+1}\right)}{\log \left(2 R_{k-2}\right)} \rightarrow 1
$$

Then quasi uniform disconnectedness follows from (2.16)-(2.19).

Lemma 3. $E^{\prime}$ is quasi Ahlfors-David s-regular. 
Proof. It is easy to check that

$$
\lim _{k \rightarrow \infty}-\frac{\log n_{1} \cdots n_{k}}{\log c_{1} \cdots c_{k}}=\lim _{k \rightarrow \infty} \frac{\log n_{1} \cdots n_{k}}{\log \left(1 / R_{k}\right)}=s .
$$

Equipping the ball $B\left(x_{i_{1} \cdots i_{k}}, R_{k}\right)$ with mass $\frac{1}{n_{1} \cdots n_{k}}$, we obtain a mass distribution $\mu$ on $F$. In order to illustrate that (2.6) holds for $F$ and $\mu$, we only need to prove that

$$
\frac{\log \mu(B(x, r))}{\log r} \rightarrow s \text { uniformly. }
$$

For this, we assume that $R_{k} / 3<r \leq R_{k-1} / 3$ and $x \in F$.

We suppose that $x \in B\left(x_{i_{1} \cdots i_{k-1} i_{k} i_{k+1}}, R_{k+1}\right)$, then $x \in B\left(x_{i_{1} \cdots i_{k-1}}, R_{k-1}\right)$. By (2.14) and $R_{k}<R_{k-1} / 6$ for small $\varepsilon$, we have $B(x, r) \subset B\left(x_{i_{1} \cdots i_{k-1}}, R_{k-1} / 2+R_{k}+r\right) \subset$ $B\left(x_{i_{1} \cdots i_{k-1}}, R_{k-1}\right)$. Thus

$$
\mu(B(x, r)) \leq \mu\left(B\left(x_{i_{1} \cdots i_{k-1}}, R_{k-1}\right)\right)=\frac{1}{n_{1} \cdots n_{k-1}} .
$$

On the other hand, since $2 R_{k+1}<R_{k} / 3(<r)$ when $\varepsilon$ is small, we have $B\left(x_{i_{1} \cdots i_{k-1} i_{k} i_{k+1}}\right.$, $\left.R_{k+1}\right) \subset B(x, r)$, which implies

$$
\mu(B(x, r)) \geq \mu\left(B\left(x_{i_{1} \cdots i_{k-1} i_{k} i_{k+1}}, R_{k+1}\right)\right)=\frac{1}{n_{1} \cdots n_{k+1}} .
$$

Therefore, we have

$$
\frac{\log n_{1} \cdots n_{k-1}}{\log \left(3 / R_{k}\right)} \leq \frac{\log \mu(B(x, r))}{\log r} \leq \frac{\log n_{1} \cdots n_{k+1}}{\log \left(3 / R_{k-1}\right)}
$$

where $\frac{\log n_{1} \cdots n_{k-1}}{\log \left(3 / R_{k}\right)}, \frac{\log n_{1} \cdots n_{k+1}}{\log \left(3 / R_{k-1}\right)} \rightarrow s$ as $k \rightarrow \infty$. Then (2.20) follows.

Since $E^{\prime}$ is quasi Ahlfors-David $s$-regular,

$$
\operatorname{dim}_{H} E^{\prime}=\operatorname{dim}_{H} E=s .
$$

Using Lemmas 1-3, we obtain Theorem 1.

\section{Regularity of Moran sets}

In this section, we consider Moran subsets of $\mathbf{R}^{1}$ generated by the initial closed interval $I$. Without loss of generality, we always assume the diameter $|I|=\left|I_{\emptyset}\right|=1$. If $\sigma=i_{1} \cdots i_{k} \in \mathcal{D}^{k}$, then each $I_{\sigma}$ is similar to $I_{\emptyset}$ with ratio $c_{1, i_{1}} \cdots c_{k, i_{k}}$ and then

$$
\left|I_{\sigma}\right|=c_{1, i_{1}} \cdots c_{k, i_{k}} .
$$

Definition 5. A Moran set $F$ defined as in (2.4) is called a homogeneous uniform Cantor set with the structure $\left(I,\left\{n_{k}\right\},\left\{c_{k}\right\}\right)$, if where $I$ is a closed interval and $\left\{c_{k}\right\}_{k \geq 1}$ is a ratios sequence such that $F$ satisfies, for all $\sigma \in \mathcal{D}^{k-1}$,

(1) $I_{\sigma * 1}, I_{\sigma * 2}, \cdots, I_{\sigma * n_{k}}$ are subintervals of $I_{\sigma * n_{k}}$, arranged from left to right;

(2) $I_{\sigma}$ and $I_{\sigma * 1}$ share left end-points, and $I_{\sigma}$ and $I_{\sigma * n_{k}}$ share right end-points;

(3) $\delta_{\sigma * 1}=\cdots=\delta_{\sigma *\left(n_{k}-1\right)}$, where $\delta_{\sigma * j}$ is the length of gap between $I_{\sigma * j}$ and $I_{\sigma *(j+1)}$.

Recall that any $I_{\sigma}$ with $\sigma \in \mathcal{D}^{k}$ is called a basic intervals of rank $k$. 
Definition 6. A compact subset $E$ of $\mathbf{R}^{n}$ is called uniformly perfect if there is a constant $0<c<1$ such that

$$
E \cap\{y: c r \leq|y-x| \leq r\} \neq \varnothing
$$

for all $0<r<|E|$ and $x \in E$.

The uniform perfectness is an interesting invariant under bilipschitz mappings $[10,20,17]$. Using the definition of regularity, we obtain the following result directly.

Lemma 4. Any Ahlfors-David regular set is uniformly perfect.

3.1. A Moran set without regular subset. We will construct a Moran set such that none of its subsets can be uniformly perfect. Then Proposition 1 follows from Lemma 4.

For any $s \in(0,1)$, let $F$ be a homogeneous uniform Cantor set with the structure $\left(I,\left\{n_{k}\right\},\left\{c_{k}\right\}\right)$, where $I_{\emptyset}=[0,1], n_{k} \rightarrow \infty, n_{k+1} / n_{k} \rightarrow \infty$ and

$$
c_{k} \equiv n_{k}^{-\frac{1}{s}} \text { for all } k>0 \text {. }
$$

Then $\operatorname{dim}_{H}(F)=\lim _{k \rightarrow \infty} \frac{\log n_{1} n_{2} \cdots n_{k}}{-\log c_{1} c_{2} \cdots c_{k}}=s$ (see [13]).

We note that the length of each gap of rank $k$

$$
\delta_{k}=\frac{1-n_{k} c_{k}}{n_{k}-1} c_{1} c_{2} \cdots c_{k-1}=\frac{1-n_{k}^{1-\frac{1}{s}}}{n_{k}-1} c_{1} c_{2} \cdots c_{k-1} .
$$

Since $n_{k+1} / n_{k} \rightarrow \infty$, we have $\delta_{k+1}<\delta_{k}$ for all $k$. Any basic interval of rank $k$ has length $\lambda_{k}=c_{1} c_{2} \cdots c_{k}$. Therefore,

$$
\lim _{k \rightarrow \infty} \frac{\lambda_{k}}{\delta_{k}}=\lim _{k \rightarrow \infty} c_{k} n_{k}=\lim _{k \rightarrow \infty}\left(n_{k}\right)^{1-\frac{1}{s}}=0 .
$$

Suppose on the contrary that $E(\subset F)$ is uniformly perfect with constant $c$ as in (3.2).

Fix a point $x \in E$. For any $k$, assume that $x$ belongs to $I_{\sigma}$ which is a basic interval of rank $k$. Note that $I_{\sigma} \subset\left\{y: \lambda_{k} \leq|x-y|\right\}$. Then the construction of $F$ implies that

$$
F \cap\left\{y: 2 \lambda_{k} \leq|x-y| \leq \delta_{k} / 2\right\}=\varnothing,
$$

which implies for all $k$,

$$
0<c \leq \frac{2 \lambda_{k}}{\delta_{k} / 2}
$$

Letting $k \rightarrow \infty$, we obtain that $c=0$. This a contradiction. Then Proposition 1 is proved.

3.2. Regular Moran set on $\mathbf{R}^{\mathbf{1}}$. We begin the proof of Proposition 2 .

" $\Longleftarrow$ Suppose $(1.5)$ holds, we will verify the regularity. In order to prove Proposition 2, we introduce the natural measure $\mu$ supported on Moran set $F$ (see to [1]). Fix $s>0$. Let

$$
\mu\left(I_{\emptyset}\right)=1,
$$

where $\emptyset$ is the empty word. By induction, for $\sigma=i_{1} \cdots i_{k} \in \mathcal{D}^{k}$, we write $\sigma^{-}=$ $i_{1} \cdots i_{k-1} \in \mathcal{D}^{k-1}$ and define

$$
\mu\left(I_{\sigma}\right)=\frac{c_{k, i_{k}}^{s}}{\sum_{j=1}^{n_{k}} c_{k, j}^{s}} \mu\left(I_{\sigma^{-}}\right) .
$$


Using (3.6) again and again, we obtain that $\mu\left(I_{\sigma}\right)=\frac{\left(c_{k, i_{k}} c_{k-1, i_{k-1}} \cdots c_{1, i_{1}}\right)^{s}}{\prod_{i=1}^{k} \sum_{j=1}^{n_{i}} c_{i, j}^{s}} \mu\left(I_{\emptyset}\right)$. By (3.1) we have

$$
\mu\left(I_{\sigma}\right)=\frac{\left|I_{\sigma}\right|^{s}}{\prod_{i=1}^{k} \sum_{j=1}^{n_{i}} c_{i, j}^{s}}
$$

More and more, we get a probability measure $\mu$ supported on $F$. Hence, by (1.5), it holds that

$$
\beta^{-1}\left|I_{\sigma}\right|^{s} \leq \mu\left(I_{\sigma}\right) \leq \alpha^{-1}\left|I_{\sigma}\right|^{s} .
$$

For any given point $x \in F$, fix $0<r \leq|F|$. The collection $\mathcal{W}_{r}$ of words is defined by

$$
\mathcal{W}_{r}=\bigcup_{k=1}^{\infty}\left\{\sigma \in \mathcal{D}^{k}: I_{\sigma} \cap B(x, r) \neq \varnothing \text { and }\left|I_{\sigma}\right| \leq r<\left|I_{\sigma^{-}}\right|\right\} \text {. }
$$

Let

$$
\mathcal{A}_{r}=\left\{I_{\sigma} \mid \sigma \in \mathcal{W}_{r}\right\} .
$$

For members of $\mathcal{A}_{r}$, since their interiors are pairwise disjoint and

$$
\left|I_{\sigma}\right| \geq c_{*} r \text { for any } I_{\sigma} \in \mathcal{A}_{r},
$$

we have $\# \mathcal{A}_{r} \leq\left(2 / c_{*}+2\right)$. Notice that

$$
B(x, r) \cap F \subset \bigcup_{I_{\sigma} \in \mathcal{A}_{r}} I_{\sigma} .
$$

According to (3.8), we have

$$
\begin{aligned}
\mu(B(x, r)) & =\mu(B(x, r) \cap F) \leq \sum_{I_{\sigma} \in \mathcal{A}_{r}} \mu\left(I_{\sigma}\right) \leq \# \mathcal{A}_{r} \cdot \max _{I_{\sigma} \in \mathcal{A}} \mu\left(I_{\sigma}\right) \\
& \leq\left(2 / c_{*}+2\right) \alpha^{-1} \cdot \max _{I_{\sigma} \in \mathcal{A}}\left|I_{\sigma}\right|^{s} \leq\left(2 / c_{*}+2\right) \alpha^{-1} \cdot r^{s}
\end{aligned}
$$

On the other hand, since $x$ is the center of $B(x, r)$, it is easy to find that there is always a word $\tau \in \mathcal{W}_{r}$ satisfying that $x \in I_{\tau}$ and $I_{\tau} \subset B(x, r)$ due to $\left|I_{\tau}\right| \leq r$. Then it holds that, by (3.8),

$$
\mu(B(x, r)) \geq \mu\left(I_{\tau}\right) \geq \beta^{-1}\left|I_{\tau}\right|^{s} \geq \beta^{-1} c_{*}^{s} r^{s} .
$$

Therefore, we can get (1.1) for the measure $\mu$ and the constant $C_{F}=\max \left\{\left(2 / c_{*}+\right.\right.$ 2) $\left.\alpha^{-1}, \beta c_{*}^{-s}\right\}$.

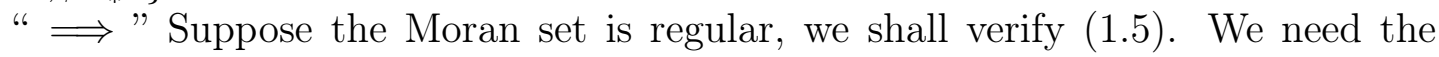
following lemma.

Lemma 5. If $F$ is $s$-regular, then there is a constant $C$ such that

$$
C^{-1}\left|I_{\sigma}\right|^{s} \leq \nu\left(I_{\sigma}\right) \leq C\left|I_{\sigma}\right|^{s}, \quad \forall \sigma \in \mathcal{D}^{\infty} .
$$

Proof. Suppose that there is a Borel probability measure $\nu$ supported on $F$ and a constant $C_{F}$ such that

$$
C_{F}^{-1} r^{s} \leq \nu(B(x, r)) \leq C_{F} r^{s} .
$$

For any given $\sigma \in \mathcal{D}^{k}$, let $\mathcal{P}$ be the set of all basic intervals of rank $(k+2)$ in $I_{\sigma}$, i.e.,

$$
\mathcal{P}=\left\{I_{\sigma * j * h}: 1 \leq j \leq n_{k+1}, 1 \leq h \leq n_{k+2}\right\}
$$


Since $n_{k} \geq 2$ for all $k>0$, it holds that $\# \mathcal{P} \geq 4$. Then we have

$$
\mathcal{Q}=\mathcal{P} \backslash\left(J_{-} \cup J_{+}\right) \neq \varnothing,
$$

where $J_{-}$is the most left member in $\mathcal{P}$ and $J_{+}$is the most right one. Moreover, it is natural that

$$
\left|J_{-}\right| \geq c_{*}^{2}\left|I_{\sigma}\right| \text { and }\left|J_{+}\right| \geq c_{*}^{2}\left|I_{\sigma}\right| .
$$

Therefore, for any one point $x \in \mathcal{Q} \cap F$, we have $B\left(x, c_{*}^{2}\left|I_{\sigma}\right|\right) \subset I_{\sigma}$. Then it holds that, by (3.15),

$$
\nu\left(I_{\sigma}\right) \geq \nu\left(B\left(x, c_{*}^{2}\left|I_{\sigma}\right|\right)\right) \geq C_{F}^{-1} \cdot c_{*}^{2 s}\left|I_{\sigma}\right|^{s}
$$

On the other hand, it is obvious that $I_{\sigma} \subset B\left(x,\left|I_{\sigma}\right|\right)$ for any $x \in F \cap I_{\sigma}$. Then

$$
\nu\left(I_{\sigma}\right) \leq \nu\left(B\left(x,\left|I_{\sigma}\right|\right)\right) \leq C_{F}\left|I_{\sigma}\right|^{s} .
$$

Therefore, let $C=\max \left\{C_{F}, c_{*}^{-2 s} C_{F}\right\}$, we have (3.14).

By (3.14), we have, $\forall k>0$,

$$
1=\nu\left(I_{\emptyset}\right)=\sum_{\sigma \in D^{k}} \nu\left(I_{\sigma}\right) \geq C^{-1} \sum_{\sigma \in D^{k}}\left|I_{\sigma}\right|^{s}=C^{-1} \prod_{i=1}^{k} \sum_{j=1}^{n_{i}} c_{i, j}^{s} .
$$

On the other hand, it is clear that

$$
1=\nu\left(I_{\emptyset}\right)=\sum_{\sigma \in D^{k}} \nu\left(I_{\sigma}\right) \leq C \sum_{\sigma \in D^{k}}\left|I_{\sigma}\right|^{s}=C \prod_{i=1}^{k} \sum_{j=1}^{n_{i}} c_{i, j}^{s} .
$$

Let $\alpha=C^{-1}$ and $\beta=C$, (1.5) holds. Then Proposition 2 follows.

3.3. An example. For $s$-regular set $E$, by Theorem 5.7 of [8], we have

$$
\overline{\operatorname{dim}}_{B} E=\underline{\operatorname{dim}}_{B} E=\operatorname{dim}_{H} E=s .
$$

For Moran set with structure $\left(J,\left\{n_{k}\right\},\left\{c_{k, j}\right\}\right)$, the positive sequence $\left\{s_{k}\right\}_{k>0}$ is called the pre-dimension sequence of $F$, where $s_{k}$ satisfies

$$
\prod_{i=1}^{k} \sum_{j=1}^{n_{i}} c_{i, j}^{s_{k}}=1
$$

Let $s_{*}=\underline{\lim }_{k \rightarrow \infty} s_{k}$ and $s^{*}=\varlimsup_{\lim _{k \rightarrow \infty}} s_{k}$. It was shown in $[13,14]$ that, if $c_{*}>0$ for Moran set $F$ as above, then

$$
\operatorname{dim}_{H} F=s_{*} \text { and } \overline{\operatorname{dim}}_{B} F=s^{*} .
$$

Therefore, if $s_{*}<s^{*}$, then $F$ can not be regular.

Example 1. Let $n_{k} \equiv 2$ and $c_{k} \in\{1 / 3,1 / 5\}$. Then $c_{*}>0$. Take a sequence $\left\{c_{k}\right\}_{k}$ such that $a=\underline{\lim }_{k \rightarrow \infty} q_{k}<\varlimsup_{k \rightarrow \infty} q_{k}=b$, where

$$
q_{k}=\frac{\#\left\{i \leq k: c_{k}=1 / 3\right\}}{k} .
$$

Then

$$
\underline{\lim }_{k \rightarrow \infty} s_{k}=\frac{\log 2}{a \log 3+(1-a) \log 5} \text { and } \varlimsup_{k \rightarrow \infty} s_{k}=\frac{\log 2}{b \log 3+(1-b) \log 5},
$$

which means $\operatorname{dim}_{H} F<\overline{\operatorname{dim}}_{B} F$ if Moran set $F$ has the structure $\left\{[0,1],\left\{n_{k}\right\},\left\{c_{k}\right\}\right\}$. Hence $F$ can not be regular. 


\section{References}

[1] DaI, Y.X., Z. X. Wen, L. XI, and Y. XIong: Quasisymmetrically minimal Moran sets and Hausdorff dimension. - Ann. Acad. Sci. Fenn. Math. 36:1, 2011, 139-151.

[2] Deng, J., Z. X. Wen, Y. Xiong, and L. F. XI: Bilipschitz embedding of self-similar sets. J. Anal. Math. 114, 2011, 63-97.

[3] K. J. FAlconer, and D. T. Marsh: On the Lipschitz equivalence of Cantor sets. - Mathematika 39, 1992, 223-233.

[4] Hutchinson, J. E.: Fractals and self-similarity. - Indiana Univ. Math. J. 30, 1981, 713-747.

[5] LI, H., Q. WANG, and L. F. XI: Classification of Moran fractals. - J. Math. Anal. Appl. 378:1, $2011,230-237$.

[6] LI, Y.Z., M. WU, and L. F. XI: Quasisymmetric minimality on packing dimension for Moran sets. - J. Math. Anal. Appl. 408:1, 2013, 324-334.

[7] Llorente, M., and P. Mattila: Lipschitz equivalence of subsets of self-conformal sets. Nonlinearity 23:4, 2010, 875-882.

[8] Mattila, P.: Geometry of sets and measures in Euclidean spaces. Fractals and rectifiability. - Cambridge Univ. Press, Cambridge, 1995.

[9] Mattila, P., and P. SaAranen: Ahlfors-David regular sets and bilipschitz maps. - Ann. Acad. Sci. Fenn. Math. 34, 2009, 487-502.

[10] Pommerenke, Ch.: Uniformly perfect sets and the Poincaré metric. - Arch. Math. 32, 1979, 192-199.

[11] WANG, Q., and L.F. XI: Quasi-Lipschitz equivalence of quasi Ahlfors-David regular sets. Sci. China Math. 54:12, 2011, 2573-2582.

[12] Wang, Q., and L. F. XI: Quasi-Lipschitz equivalence of Ahlfors-David regular sets. - Nonlinearity 24:3, 2011, 941-950.

[13] Wen, Z. Y.: Moran sets and Moran classes. - Chinese Sci. Bull. 46:22, 2001, 1849-1856.

[14] Wen, Z. Y.: Mathematical foundation of fractal geometry. - Shanghai Scientific and Technological Education Publishing House, Shanghai, 2000.

[15] Wen, Z. Y., and L.F. XI: Relations among Whitney sets, self-similar arcs and quasi-arcs. Israel J. Math. 136, 2003, 251-267.

[16] Wen, Z. Y., and L.F. XI: On the dimensions of sections for the graph-directed sets. - Ann. Acad. Sci. Fenn. Math. 35:2, 2010, 515-535.

[17] XI, L. F.: Lipschitz equivalence of self-conformal sets. - J. London Math. Soc. (2) 70:2, 2004, $369-382$.

[18] XI, L. F.; Quasi-Lipschitz equivalence of fractals. - Israel J. Math. 160, 2007, 1-21.

[19] XI, L. F.: Lipschitz equivalence of dust-like self-similar sets. - Math. Z. 266:3, 2010, 683-691.

[20] XIE, F., Y. C. YIN, and Y. S. Sun: Uniform perfectness of self-affine sets. - Proc. Amer. Math. Soc. 131, 2003, 3053-3057.

Received 7 October 2013 • Accepted 17 December 2013 